\title{
A Fully-integrated Split-electrode SSHC Rectifier for Piezoelectric Energy Harvesting
}

\author{
Sijun Du, Member, IEEE, Yu Jia, Member, IEEE, Chun Zhao, Member, IEEE, Gehan A. J. Amaratunga, \\ and Ashwin A. Seshia, Senior Member, IEEE
}

\begin{abstract}
In order to efficiently extract power from piezoelectric vibration energy harvesters, various active rectifiers have been proposed in the past decade, which include Synchronized Switch Harvesting on Inductor (SSHI), Synchronous Electric Charge Extraction (SECE), etc. Although reported active rectifiers show good performance improvements compared to fullbridge rectifiers (FBR), large off-chip inductors are typically required and the system volume is inevitably increased as a result, counter to the requirement for system miniaturization. In this paper, a fully-integrated split-electrode SSHC (synchronized switch harvesting on capacitors) rectifier is proposed, which achieves significant performance enhancement without employing any off-chip components. The proposed circuit is designed and fabricated in a $0.18 \mu \mathrm{m}$ CMOS process and it is co-integrated with a custom MEMS (microelectromechanical systems) piezoelectric transducer with its electrode layer equally split into four regions. The measured results show that the proposed rectifier can provide up to $8.2 \times$ and $5.2 \times$ boost, using on-chip and off-chip diodes respectively, in harvested power compared to a FBR under low excitation levels and the peak rectified output power achieves $186 \mu \mathrm{W}$.
\end{abstract}

Index Terms-Energy harvesting, piezoelectric transducer, synchronized switch harvesting on inductor (SSHI), synchronized switch harvesting on capacitors (SSHC), fully-integrated system, switched capacitors, power conditioning, rectifiers.

\section{INTRODUCTION}

Along with the development of the Internet of Everything (IoE), miniaturized piezoelectric vibration-based energy harvesters have drawn significant interest as a means of harvesting ambient kinetic energy to power wireless sensor nodes in wireless sensor networks (WSN) [1]-[3]. Since the environmental vibration is periodic and highly unpredictable, the energy generated by a piezoelectric transducer (PT) cannot be directly used and an interface circuit is needed to rectify the generated power and provide a stable DC supply to the loads. Full-bridge rectifiers (FBR) are widely employed in most commercially available power management units (PMU)

S. Du is with the Department of Engineering, University of Cambridge, Cambridge, CB2 1PZ, UK, and also with the Department of Electrical Engineering and Computer Sciences, University of California at Berkeley, Berkeley, CA, 94720, USA. (e-mail: sd672@cam.ac.uk; sijun@eecs.berkeley.edu).

Y. Jia is with the Department of Mechanical Engineering, University of Chester, Chester, CH2 4NU, UK, and also with the Department of Engineering, University of Cambridge, Cambridge, CB2 1PZ, UK.

C. Zhao is with the Department of Engineering, University of Cambridge, Cambridge, CB2 1PZ, UK, and also with the Center for Gravitational Experiments, Huazhong University of Science and Technology, Wuhan, 430074, China.

G. A. J. Amaratunga and A. A. Seshia are with the Department of Engineering, University of Cambridge, Cambridge, CB2 1PZ, UK.

Correspondence and requests for materials should be addressed to Ashwin A. Seshia (e-mail: aas41@cam.ac.uk). due to their simplicity and stability; however, the low powerextraction efficiency of FBRs limits the usable output power for loads, especially under low ambient excitation levels [4], [5]. In order to increase rectified power, a variety of active interface circuits have been proposed in the past decades, which include SSHI (synchronized switch harvesting on inductor), SECE (synchronous electric charge extraction), etc [6]-[16]. Although these reported rectifiers show good performance enhancement compared to FBRs under low excitation levels, they typically require large inductors to achieve good performance and the inductance values can be up to 10 's $\mathrm{mH}$. These inductors significantly increase the system volume, especially for MEMS-CMOS co-integrated systems, counter to the requirement for system miniaturization.

Recently, an inductorless interface circuit was proposed by Chen et al. [17] that uses a technique called flippingcapacitor rectifier (FCR), which employs a number of switched capacitors (SC) to synchronously flip the voltage across the PT. The work achieves a fully integrated implementation with high performance enhancement thanks to the small inherent capacitance of the PT $\left(C_{P}=80 \mathrm{pF}\right)$. The term "high performance" used here and later in this paper means the extracted power ratio between using an active rectifier and using a passive FBR is high, and this ratio is usually in range of $1-10$ for reported interface circuits. However, this small PT with high resonant frequency $(110 \mathrm{kHz})$ used in [17] is typically used in ultrasonic wireless power transfer (WPT) systems and may not be suitable for kinetic vibration energy harvesting since the real-world kinetic vibration typically has a much lower frequency range between 10 's $\mathrm{Hz}$ and 1's $\mathrm{kHz}$. PTs targeting this frequency range usually have much higher inherent capacitance $C_{P}$ (between 1's $\mathrm{nF}$ and 100's $\mathrm{nF}$ ), and as a result, the required total capacitance value for the FCR technique will be too large to be implemented on-chip. Besides the FCR technique, a SSHC (synchronized switch harvesting on capacitors) rectifier has been proposed in [18]. Similar to SSHI rectifiers, the SSHC rectifier employs capacitors instead of inductors to perform synchronous voltage flip. The number of employed capacitors can be configured to any positive integer according to the trade-off on performance and system volume: more capacitors result in higher performance.

Fig. 1 shows the circuit diagrams of a full-bridge rectifier, a SSHI rectifier and a SSHC rectifier. While the PT is vibrating, it can be modeled as a current source $I_{P}$ in parallel with a capacitor $C_{P}$. This equivalent circuit model of a PT has been proven to be inaccurate for PTs with strong electromechanical coupling [19]. However, the PT used in this paper is fabricated 


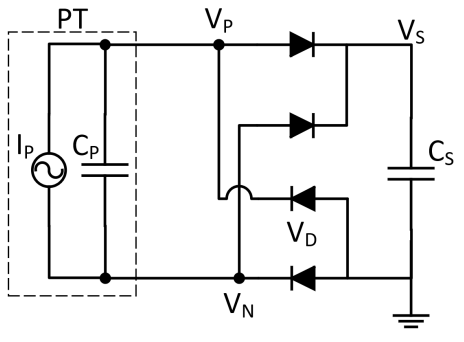

(a) Full-bridge rectifier.

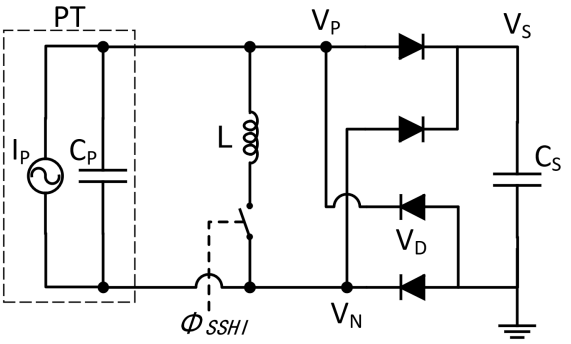

(b) SSHI rectifier.
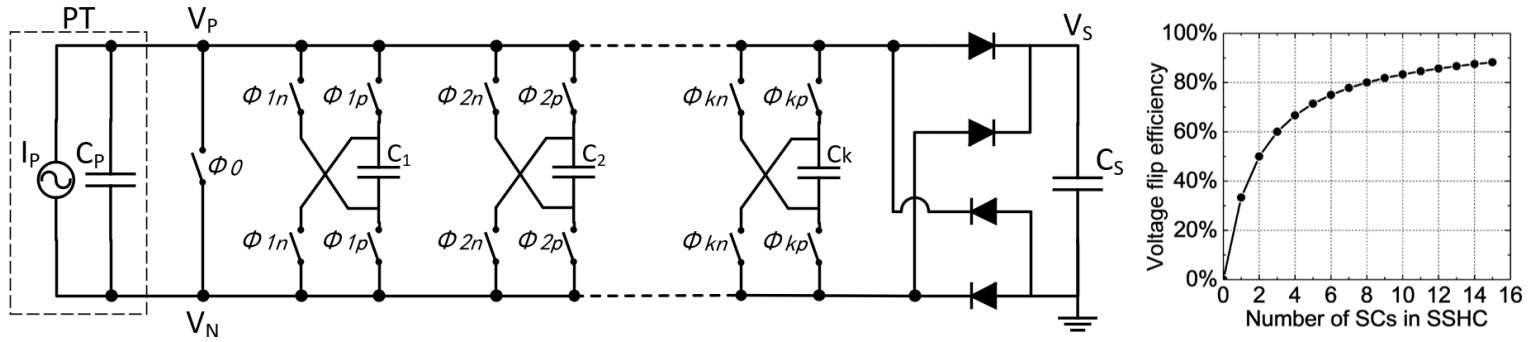

(c) SSHC rectifier with $k$ switched capacitors (SC) and the theoretical voltage flip efficiencies.

Fig. 1: Circuit diagrams of (a) a full-bridge rectifier, (b) a SSHI rectifier and (c) a SSHC rectifier with $k$ switched capacitors.

in a commercial MEMS process with AIN (Aluminum Nitride) as the piezoelectric material. Due to the low piezoelectric coefficient and coupling factor of AIN (around $1-2$ orders of magnitude lower than PZT), the electromechanical coupling is weak for AlN and the generated voltage across the PT has very little effect on the mechanical vibrations. In this case, the specified equivalent circuit model comprising a current source and a capacitor is sufficiently accurate. The voltage flip efficiency plot in Fig. 1c shows that more SCs in a SSHC rectifier can increase the voltage flip efficiency, hence, the power extraction performance. The relation between voltage flip efficiency and output power will be derived later in Section II-B. Previous work clarifying this relation can also be found in [6], [16], [20], [21]. According to different applications and performance requirements, the SSHC rectifier can be configured by employing a suitable number of SCs. Despite the performance and configurable architecture of the SSHC rectifier, the switched capacitors (SC) are sometimes difficult to be implemented on-chip, since the SCs need to have equal capacitance as $C_{P}$ to achieve optimal performance [18]. The term "optimal performance" used here and later in this paper means the voltage flip efficiency is maximum while varying the capacitance values of the SCs. This peak is achieved while $C_{P}$ is the same as all the SCs and the flip efficiency plot in Fig. 1c actually shows the optimal flip efficiency when $C_{P}=C_{S C}$. Although on-chip implementations can be easily achieved while using small PTs, such as the PT with $C_{P}=80 \mathrm{pF}$ used in [17], it is almost impossible to implement the SCs on-chip for large PTs in kinetic vibration energy harvesting systems since such PTs typically have $C_{P}$ values between 1 's $\mathrm{nF}$ and 100 's nF.

In this paper, a fully integrated split-electrode SSHC (SE$\mathrm{SSHC}$ ) rectifier is proposed to perform synchronous voltage flip. With the proposed architecture, required SCs to achieve the optimal performance is significantly reduced so that they

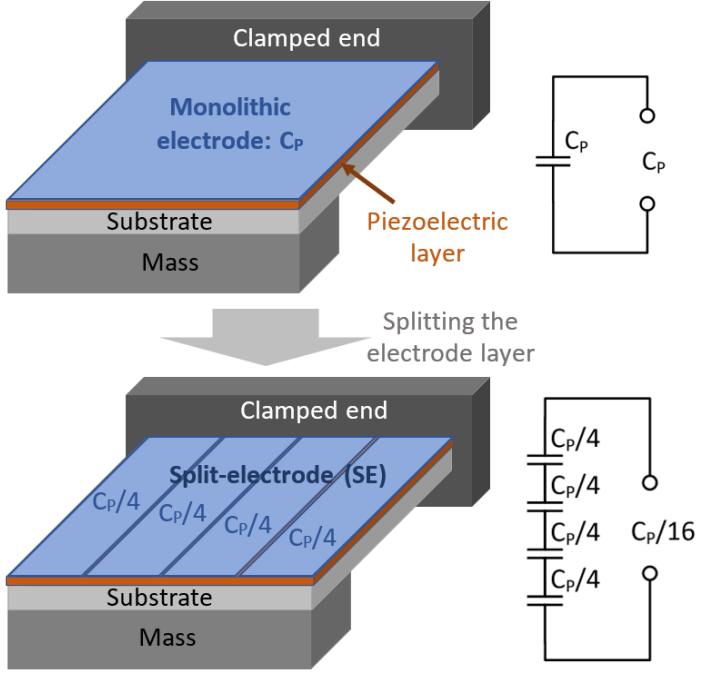

Fig. 2: Splitting the monolithic electrode electrode of a PT into 4 regions.

can be easily implemented on-chip even for PTs with large $C_{P}$ capacitance. Since this design does not require any off-chip component (except for power capacitors), the system volume is significantly reduced while achieving high performance. The rest of this paper is organized as follows. Section II presents the proposed SE-SSHC rectifier and provides power analysis in comparison with a FBR. Detailed circuit implementations are shown in Section III. Measured results and comparisons with state-of-the-art rectifiers are provided in Section IV and a conclusion is provided in the last section.

\section{PROPOSED SE-SSHC SYSTEM}

As previously discussed, the SSHC rectifier shown in Fig. 1c requires all the switched capacitors $(\mathrm{SC})$ have the equal 


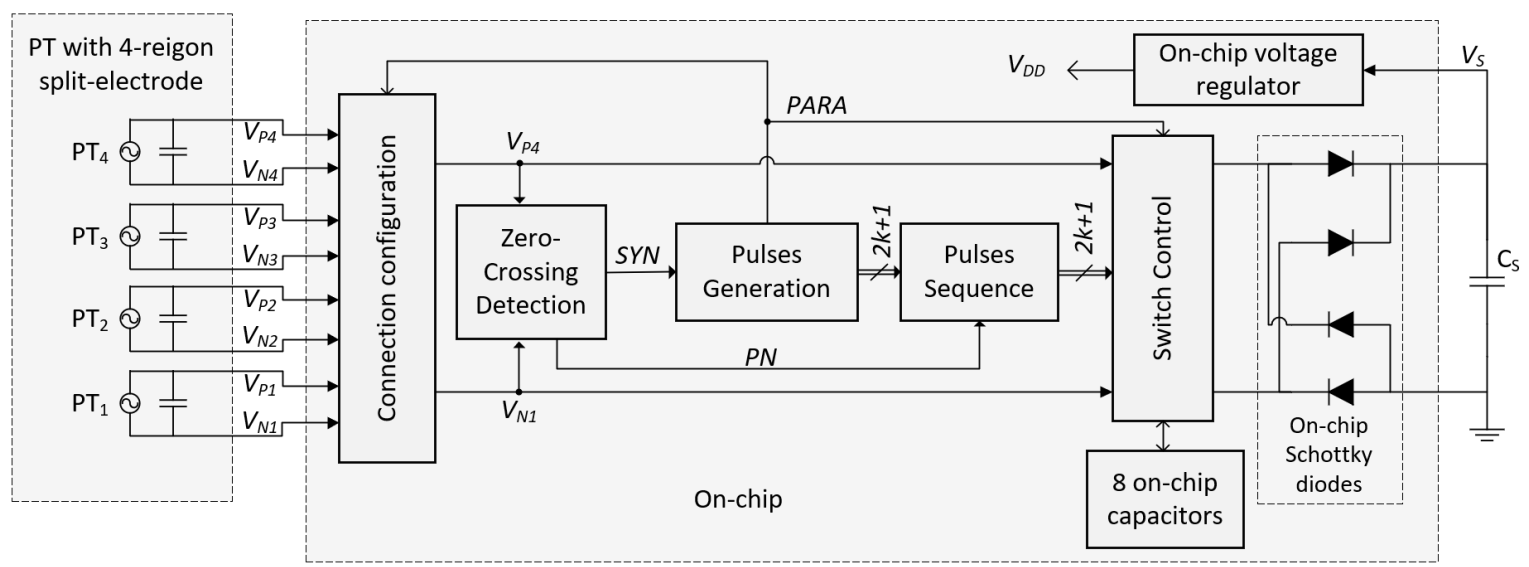

Fig. 3: System architecture of the proposed SE-SSHC rectifier.

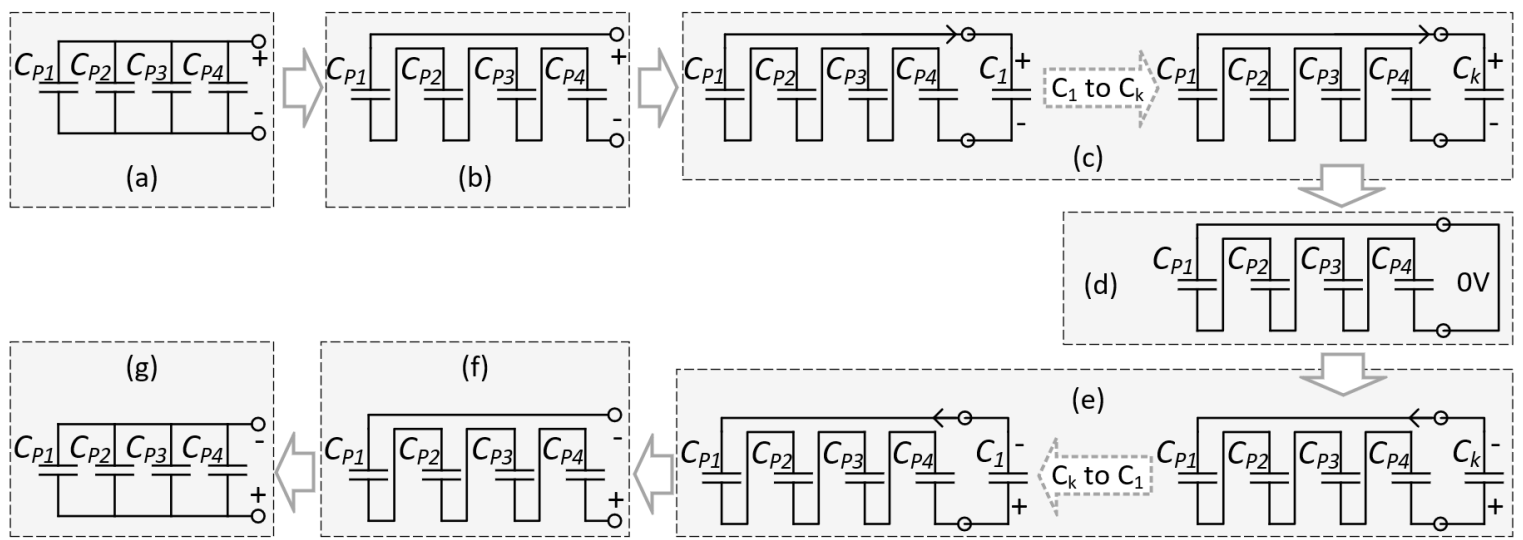

Fig. 4: Schematic description of the connection configuration cycle during voltage flip.

capacitance as that of the PT to achieve the optimal performance, such as $C_{1}=C_{2}=\cdots=C_{k}=C_{P}$. This requirement makes on-chip implementation of SCs very impractical when PTs with large $C_{P}$ values are employed. In order to address a fully integrated implementation, the optimal SC values need to be significantly decreased. In the proposed SE-SSHC system, a split-electrode PT is employed with its electrode layer split into several regions, assuming $n$ regions $(n=4$ in this work). During the voltage flip time instants, the four regions are temporarily connected in series so that the effective capacitance of the PT is decreased by $4^{2}=16$ times, as shown in Fig. 2. As a result, the required SCs in the SSHC rectifier are also decreased by 16 times such that they can be implemented on-chip. In this work, the PT is designed and fabricated in a commercial MEMS process. In order to achieve this design, the $n$ regions need to be completely separated for all the layers in the MEMS process, including the top electrode layer, piezoelectric layer and doped-Silicon substrate layer. These $n$ regions are strongly coupled with a common proof mass at the free end of the cantilever. With this design, the $n$ regions are mechanically coupled and electrically insulated. As a result, the signals generated from all the regions are identical in amplitude, frequency and phase; hence, they can be perfectly connected in parallel or in series [22]. This section presents the proposed SE-SSHC rectifier at the system level.
The performance compared with a FBR is then theoretically analyzed.

\section{A. System architecture}

Fig. 3 shows the system architecture of the proposed SESSHC rectifier co-integrated with a PT with its electrode split into 4 regions. The regions of the PT are connected into the SE-SSHC system through the "connection configuration" block, which configures the connection of the four regions according to the signal $P A R A$. At each zero-crossing moment of the current source generated from the PT, the "zero-crossing detection" block generates a rising edge in the signal $S Y N$ by monitoring voltages at nodes $V_{P 4}$ and $V_{N 1}$. A signal $P N$ is also generated indicating the polarization of the voltage across the PT, noted as $V_{P T}$, which equals to $V_{P 4}-V_{N 1}$. Following the rising edge of $S Y N, 2 k+1$ pulses are sequentially generated, where $k$ is the number of SCs enabled for a SSHC rectifier. In the meantime, the signal $P A R A$ is pulled down to force a series connection of the 4 electrode regions and to disconnect the system from the full-bridge rectifier (FBR) during the voltage flip instants. The following "pulse sequence" block sequences the $2 k+1$ pulses according to the signal $P N$ to flip $V_{P T}$ from positive to negative, or viceversa. The sequenced $2 k+1$ pulses drive the "switch control" block together with the on-chip SCs to perform voltage flip. 


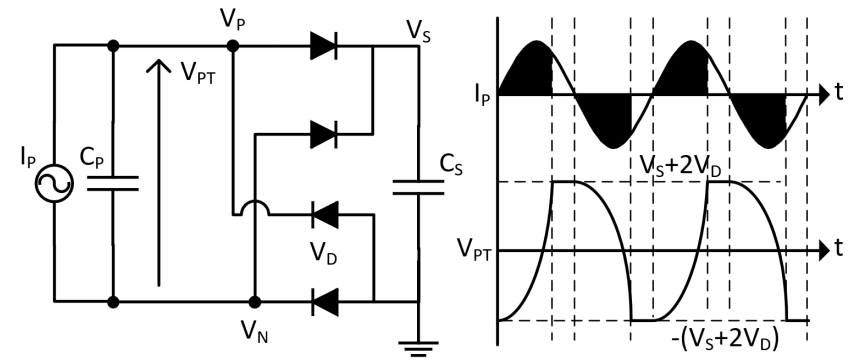

Fig. 5: Full-bridge rectifier and the associated waveforms.

After the voltage flip is complete, $P A R A$ goes back to high to configure the 4 regions back to a parallel connection until the next zero-crossing moment. An on-chip voltage regulator is employed to generate a $1.5 \mathrm{~V} \mathrm{DC}$ supply for the system.

Fig. 4 shows one connection configuration cycle while $V_{P T}$ is being flipped from positive to negative. State (a) is the moment to commence the voltage flip, which is also the zerocrossing moment of $I_{P}$. Before voltage flip commences, the four regions of the PT is configured to be connected in series, as shown in (b). The states (c), (d) and (e) show how the voltage is flipped with $2 k+1$ sequential pulses, where $k$ is the number of SCs enabled in a SSHC rectifier. The first $k$ pulses connect the PT to all the $k$ SCs sequentially from $C_{1}$ to $C_{k}$. The middle pulse shorts the PT to clear the remaining charge in the PT. The last $k$ pulses connect the $k$ SCs to the PT sequentially, but in a reversed order (from $C_{k}$ to $C_{1}$ ) and in an opposite polarization compared to the first $k$ pulses. After all these $2 k+1$ pulses, the voltage across the PT is flipped, as shown in (f). The four regions of the PT is then configured back to a parallel connection shown in $(\mathrm{g})$. When the voltage across the PT needs to be flipped from negative to positive, the configuration cycle is reversed to be from state $(\mathrm{g})$ to state (a).

\section{B. Performance analysis}

In order to evaluate the performance enhancement of the proposed circuit, it is useful to analyze the rectified power of a passive full-bridge rectifier (FBR). Fig. 5 shows the circuit diagram of a FBR and the associated waveforms. When the PT is vibrating, it can be modeled as a current source, $I_{P}$, in parallel with a capacitor, $C_{P}$. The current source can be expressed as $I_{P}=I_{0} \sin (\omega t)$, where $\omega=2 \pi f_{P}$ and $f_{P}$ is the excitation frequency. In a half period of $I_{P}$, the total generated charge by the PT can be expressed as:

$$
Q_{t o t}=\int_{0}^{\frac{T}{2}} I_{0} \sin \omega t \mathrm{~d} t=\frac{2 I_{0}}{\omega}
$$

While the PT is in an open-circuit, the open-circuit zero-topeak amplitude can be shown as:

$$
V_{O C}=\frac{1}{2} \frac{Q_{t o t}}{C_{P}}=\frac{I_{0}}{\omega C_{P}}
$$

When the PT is connected to a FBR, the FBR sets a voltage threshold for $V_{P T}$ to overcome and $V_{P T}$ needs to attain either $V_{S}+2 V_{D}$ or $-\left(V_{S}+2 V_{D}\right)$ before generated charge can be

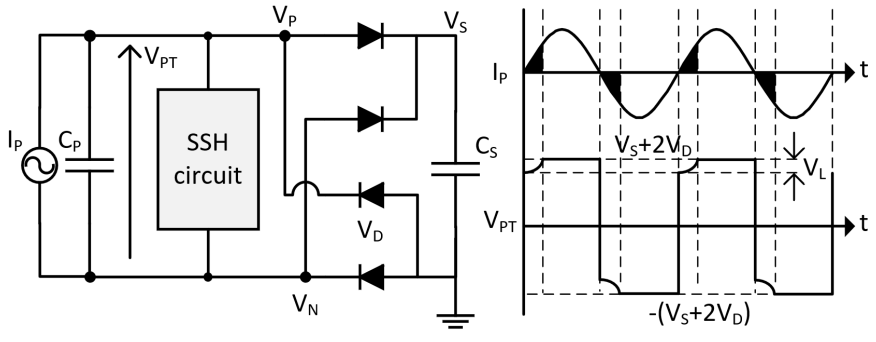

Fig. 6: SSH rectifier and the associated waveforms.

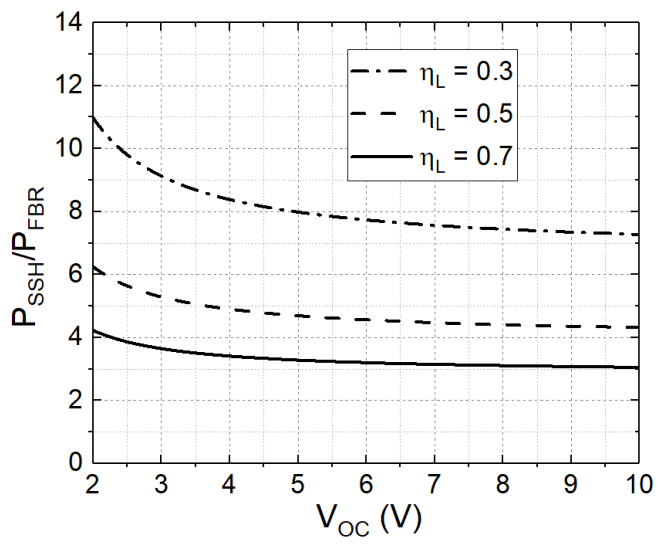

Fig. 7: Theoretical performance improvement of a SSH rectifier compared with a FBR $\left(P_{S S H} / P_{F B R}\right)$ with on-chip diodes $\left(V_{D}=0.25 \mathrm{~V}\right)$ in a range of $V_{O C}$.

transferred into the power capacitor $C_{S}$, where $V_{S}$ is the voltage across the capacitor $C_{S}$ and $V_{D}$ is the forward voltage drop of the diodes. For each half period, $V_{P T}$ needs to be flipped from $\pm\left(V_{S}+2 V_{D}\right)$ to $\mp\left(V_{S}+2 V_{D}\right)$ and the charge used to flip $V_{P T}$ between these two voltage levels is not extracted. Hence, the remaining charge transferred into $C_{S}$ can be written as:

$$
\begin{aligned}
Q_{F B R} & =Q_{t o t}-2\left(V_{S}+2 V_{D}\right) C_{P} \\
& =2 C_{P}\left(V_{O C}-V_{S}-2 V_{D}\right)
\end{aligned}
$$

Assuming the voltage increase in $C_{S}$ in a half $I_{P}$ period is very small compared to $V_{S}$ value, the extracted power stored in $C_{S}$ can be expressed as:

$$
P_{F B R}=\frac{V_{S} Q_{F B R}}{T / 2}=4 f_{P} C_{P} V_{S}\left(V_{O C}-V_{S}-2 V_{D}\right)
$$

The peak value of $P_{F B R}$ can be found by setting the derivative of (4) to 0 and the optimal value of $V_{S}$ to achieve the peak power is:

$$
V_{S, o p t}=\frac{V_{O C}}{2}-V_{D}
$$

While $V_{S}$ achieves its optimal value shown in (5), the peak extracted power by a FBR can be expressed as:

$$
P_{F B R, \max }=4 f_{P} C_{P}\left(\frac{V_{O C}}{2}-V_{D}\right)^{2}
$$




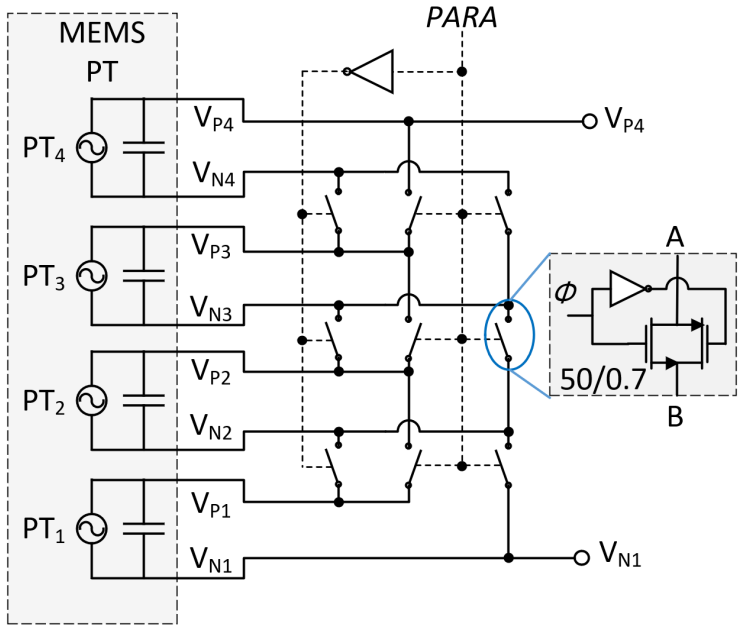

Fig. 8: Circuit diagram of the connection configuration block.

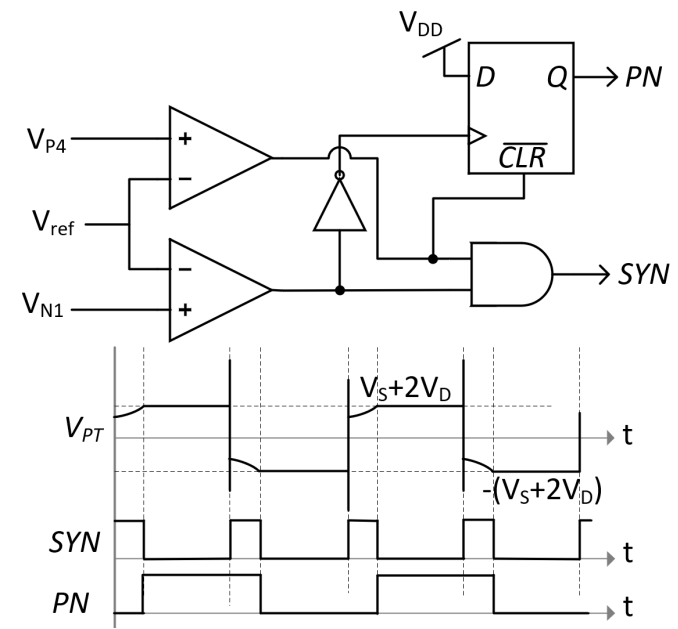

Fig. 9: Circuit diagram of the zero-crossing detection block.

The power expressed in (6) is the peak power while $V_{S}=V_{S, o p t}$. While off-chip Schottky diodes are employed, the effective voltage drop $V_{D}$ at the current level of $I_{P}$ can be very low and $V_{D}$ can be ignored in this case. However, onchip Schottky diodes usually have higher $V_{D}$ values and the voltage drop cannot be ignored for fully integrated systems.

The extracted power of a SSH (synchronized switch harvesting) rectifier will also be analyzed to show the improvement compared with the FBR. Fig. 6 shows the circuit diagram of a SSH rectifier and the associated waveforms. A SSH rectifier aims to synchronously flip the voltage $V_{P T}$ every half period of $I_{P}$ to minimize the charge wastage due to flipping $V_{P T}$ with the generated charge by the PT. However, there is some voltage loss after voltage flip and it is shown as $V_{L}$ in the waveform of $V_{P T}$. The loss ratio after voltage flip can be written as:

$$
\eta_{L}=\frac{V_{L}}{V_{S}+2 V_{D}}
$$

Some amount of charge generated by the PT is used to compensate the loss $V_{L}$; hence, the remaining charge transferred into $C_{S}$ can be written as:

$$
Q_{S S H}=Q_{t o t}-V_{L} C_{P}=C_{P}\left(2 V_{O C}-V_{L}\right)
$$

Therefore, the extracted power stored in $C_{S}$ can be expressed as:

$$
P_{S S H}=\frac{V_{S} Q_{S S H}}{T / 2}=2 f_{P} C_{P} V_{S}\left(2 V_{O C}-V_{L}\right)
$$

The above equation attains its maximum power while $V_{S}$ satisfies:

$$
V_{S, o p t}=\frac{V_{O C}}{\eta_{L}}-V_{D}
$$

The maximum output power of a SSH rectifier can then be written as:

$$
P_{S S H, \max }=2 \eta_{L} f_{P} C_{P}\left(\frac{V_{O C}}{\eta_{L}}-V_{D}\right)^{2}
$$

Comparing the peak power values for a FBR in (6) and a $\mathrm{SSH}$ rectifier in (11), the performance enhancement of a SSH rectifier can be expressed as:

$$
\frac{P_{S S H, \text { max }}}{P_{F B R, \max }}=\frac{2}{\eta_{L}}\left(\frac{V_{O C}-\eta_{L} V_{D}}{V_{O C}-2 V_{D}}\right)^{2}
$$

The equation (12) is plotted in Fig. 7 with $V_{D}=0.25 \mathrm{~V}$ (onchip diodes). The three lines represent values for three voltage flip loss ratios of $\eta_{L}=0.3, \eta_{L}=0.5$ and $\eta_{L}=0.7$, which are approximately equal to measured values while enabling 8,3 and $1 \mathrm{SCs}$, respectively, in the proposed SE-SSHC rectifier in Section IV. It can be seen that $P_{S S H} / P_{F B R}$ is higher when loss ratio is lower (or flip efficiency is higher). If discrete Schottky diodes are employed, the voltage drop $V_{D}$ can be ignored and the above becomes $2 / \eta_{L}$. It can be seen that the performance enhancement of a SSH rectifier depends on the voltage flip efficiency (or loss ratio $\eta_{L}$ ) and the loss ratio can be decreased by employing larger inductors in SSHI rectifiers or employing more SCs in SSHC rectifiers.

\section{Circuit implementations}

From the system architecture shown in Fig. 3, it can be seen that there are five main blocks in the proposed SE-SSHC interface circuit, which are the "connection configuration", "zerocrossing detection", "pulses generation", "pulses sequence" and "switch control" (including 8 on-chip configurable SCs) blocks. The detailed transistor-level circuit diagrams and operations for all the blocks are presented in the following sections.

\section{A. Connection configuration}

Fig. 8 shows the circuit diagram of the connection configuration block, which connects the four regions of the PT into the SE-SSHC rectification system. Since the four regions need to be connected in series during voltage flip moments, this block configures the connection of the PT according to the signal PARA. While PARA is high, the four regions are electrically connected in parallel, otherwise, in series. During the series connection, the four regions should be connected 


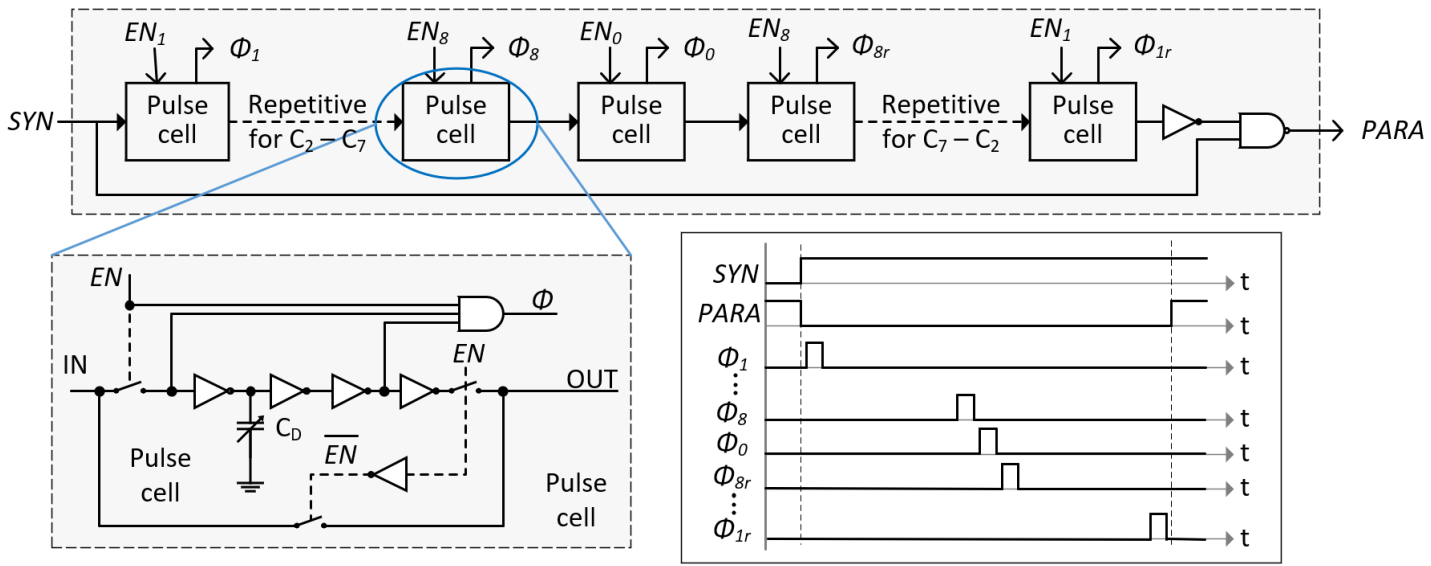

Fig. 10: Circuit diagram of the pulse generation block and associated waveforms.

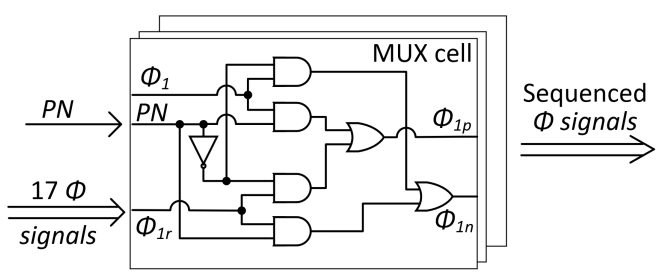

Fig. 11: Circuit diagram of the pulse sequencing block.

with consideration of voltage polarity of each region so that the series-connected model results in a summed-up voltage. Regardless of which connection configuration is chosen, nodes $V_{P 4}$ and $V_{N 1}$ are always connected to the system.

\section{B. Zero-crossing detection}

The circuit diagram and associated waveforms of the zerocrossing detection block is shown in Fig. 9. The voltage flip occurs while the current source generated from the PT is close to zero, which is also the moment when $V_{P T}$ achieves its peak value, where $V_{P T}=V_{P 4}-V_{N 1}$. In order to find this moment, two continuous-time comparators are employed to compare $V_{P 4}$ and $V_{N 1}$ with a reference voltage $V_{\text {ref }}$. While $V_{P T}$ achieves its peak and starts to rise or decrease, one of $V_{P 4}$ and $V_{N 1}$ is close to $-V_{D}$ and the other one is close to $V_{S}+V_{D}$. The reference voltage $V_{r e f}$ is set slightly higher than the negative value of the voltage drop of a diode $\left(-V_{D}\right)$ so that one of the two comparators can be triggered when either $V_{P 4}$ or $V_{N 1}$ leaves $-V_{D}$ and starts to increase towards $V_{S}+V_{D}$. At this moment, a rising edge is generated in the signal $S Y N$ and it keeps high until the voltage $V_{P T}$ is completed flipped. In this block, the signal $P N$ is also generated to indicate the polarization of $V_{P T}$ at the moment of the rising edge of $S Y N$. This signal is used in following blocks to sequence up to 17 pulses according to the voltage flip direction, from $\pm\left(V_{S}+\right.$ $\left.2 V_{D}\right)$ to $\mp\left(V_{S}+2 V_{D}\right)$.

\section{Pulse generation and sequencing}

Fig. 10 shows the circuit diagram and waveforms of the pulse generation block. In this implementation, the number of employed on-chip SCs in the SSHC rectifier is chosen at $k=8$; hence, up to $17(2 k+1)$ pulses should be generated to perform voltage flip. In this block, 17 pulse cells are employed to generate up to 17 non-overlapping sequential pulses. At each rising edge of the input signal $S Y N$, the 17 pulse cells are sequentially driven to generate one individual pulse in each cell. The 8 on-chip SCs can be selectively enabled by eight input signals $E N_{1}-E N_{8}$ and the pulse $\phi_{0}$ is enabled by $E N_{0}$. While all of the $9 E N$ signals are low, the entire system simply works as a full-bridge rectifier (FBR) since all of the switches in the SSHC rectifier keep OFF. While only $E N_{0}$ is enabled, the system works as a switch-only rectifier, which has been presented in [6]. The input $E N_{0}$ is forced to high if any of $E N_{1}-E N_{8}$ are high because the phase $\phi_{0}$ is indispensable in a SSHC rectifier to clear the residual charge in $C_{P}$ in the middle phase of the voltage flip process. Besides the up to 17 pulses, the signal $P A R A$ keeps low during the entire voltage flip process to configure the four regions of the PT to a series connection. The circuit diagram of a pulse cell is also presented in the figure and it shows that one individual pulse is generated by ANDing the delayed and inverted version of the input signal. All of the 17 pulse cells are identical and the input of each cell is the output of the previous cell, except that the input of the first cell is $S Y N$. The delay in one cell is performed using two weak inverters charging an on-chip capacitor, which can be adjusted to tune the pulsewidth of the generated pulse signal. The three switches in the pulse cell aim to enable and bypass the selected cells according to the $E N$ signals.

Before the 17 pulses are used to drive the switches to perform voltage flip, they need to be sequenced according to the voltage flip directions, which is the signal $P N$. When $P N$ is high, the voltage across the PT is positive and it needs to be flipped towards negative; hence, the sequence of the 17 pulses should be $\phi_{1 p} \rightarrow \phi_{2 p} \rightarrow \phi_{3 p} \rightarrow \phi_{4 p} \rightarrow \phi_{5 p} \rightarrow \phi_{6 p} \rightarrow \phi_{7 p} \rightarrow$ $\phi_{8 p} \rightarrow \phi_{0} \rightarrow \phi_{8 n} \rightarrow \phi_{7 n} \rightarrow \phi_{6 n} \rightarrow \phi_{5 n} \rightarrow \phi_{4 n} \rightarrow \phi_{3 n} \rightarrow$ $\phi_{2 n} \rightarrow \phi_{1 n}$. The first 8 pulses aim to sequentially transfer charge from the PT to the $8 \mathrm{SCs}, C_{1}$ to $C_{8}$. The middle pulse $\phi_{0}$ clears the residual charge in the PT and the following 8 pulses sequentially connect the $8 \mathrm{SCs}$ in an opposite sense to 


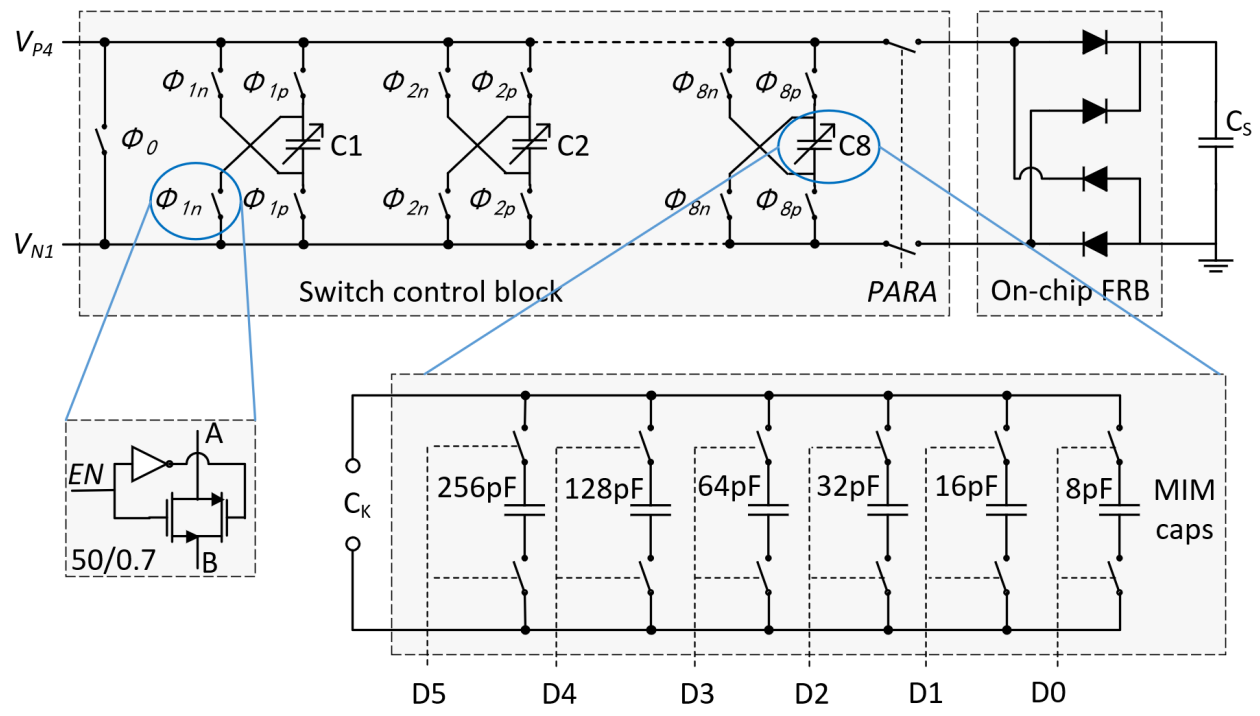

Fig. 12: Circuit diagram of the switch control block and on-chip switched capacitors.

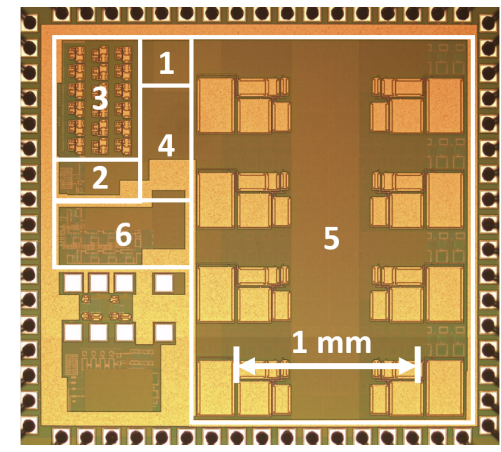

Fig. 13: Micrograph of the test chip fabricated in a $0.18 \mu \mathrm{m}$ CMOS foundry process. The active area of the proposed SESSHC rectifier is around $3.9 \mathrm{~mm}^{2} .1$, zero-crossing; 2 , connection configuration; 3 , pulses generation; 4 , pulses sequence; 5 , switch control; 6, on-chip FBR and voltage regulator.

flip the voltage $V_{P T}$ towards negative. When $P N$ is low, the voltage $V_{P T}$ needs to flipped from negative to positive and the order of the 17 pulses should be completely reversed. Fig. 11 shows the circuit diagram of the pulse sequencing block, which consists of 9 identical MUX (multiplexer) cells. 8 MUX cells are for 16 pulses driving SCs from $C_{1}$ to $C_{8}$ and a redundant MUX cell is added for the pulse $\phi_{0}$ to ensure that all pulses have the same delay to avoid overlapping.

\section{Switch control and on-chip capacitor arrays}

Fig. 12 shows the circuit diagram of the switch control block, which consists of 35 analog CMOS switches in total, including 1 switch for $\phi_{0}, 32$ switches for the 8 SCs and 2 additional switches to disconnect the system from the FBR during voltage flip instant. The eight SCs, $C_{1}-C_{8}$, are implemented on-chip with 8 dual-MIM capacitor arrays and their capacitance can be adjusted externally according to the capacitance of the PT, $C_{P}$. Since the electrode of the PT is split into 4 regions in this implementation, the resulting
TABLE I: Power consumption breakdown.

\begin{tabular}{ccc}
\hline \hline Loss mechanism & Power loss & Percentage \\
\hline Connection config & $390 \mathrm{nW}$ & $15.1 \%$ \\
Zero-cross detection & $221 \mathrm{nW}$ & $8.5 \%$ \\
Pulse generation & $85 \mathrm{nW}$ & $3.3 \%$ \\
Pulse sequencing & $0.4 \mathrm{nW}$ & $0.02 \%$ \\
Switch control & $1470 \mathrm{nW}$ & $56.8 \%$ \\
Voltage regulator & $423 \mathrm{nW}$ & $16.3 \%$ \\
Simulated total & $2.59 \mu \mathrm{W}$ & $100 \%$ \\
\hline Measured total & $\sim 0.9 \mu \mathrm{W}$ & (static) \\
Measured total & $\sim 2.9 \mu \mathrm{W}$ & (dynamic) \\
\hline \hline
\end{tabular}

SC value should equal to $C_{P} / 16$ for optimal voltage flip performance. In this work, the capacitor array for one SC can be set up to around $500 \mathrm{pF}$; hence, the system can support PTs with $C_{P}$ capacitance as high as $8 \mathrm{nF}$, which include most MEMS (microelectromechanical systems) PTs and even some macroscopic PTs. Since the smallest capacitance of the SCs can be set to $8 \mathrm{pF}$, which corresponds to $C_{P}=64 \mathrm{pF}$, the proposed system can also support most piezoelectric ultrasonic power receivers with $C_{P}$ between 10's -100 's $\mathrm{pF}$. The density of the dual-MIM capacitor in this implementation is $4.1 \mathrm{fF} / \mu \mathrm{m}^{2}$. Main parasitic factors in this block include the parasitic capacitance added into the capacitor array and the parasitic resistance of the switches. The former introduces additional capacitance in the capacitor array resulting in a value of capacitance greater than the expected value for an optimal voltage flip efficiency. The latter increases the time constant for charging and discharging the SCs. However, due to the small capacitance of SCs, thanks to the split-electrode design, and the low operating frequency (100's Hz), the switch resistance has little effect on the performance.

\section{Measurement Results}

The proposed SE-SSHC interface circuit was designed and fabricated in a $0.18 \mu \mathrm{m}$ HV CMOS process. The die photo of 


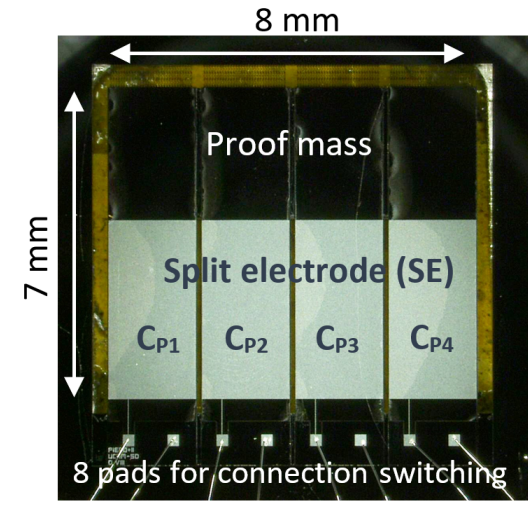

(a) PT fabricated in MEMS process by MEMSCAP.

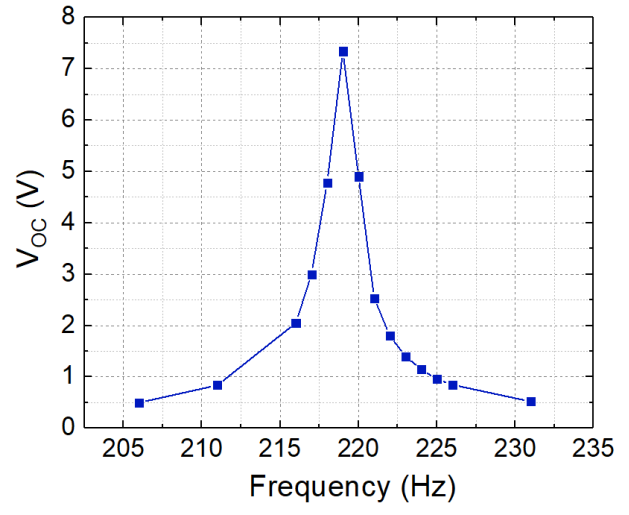

(b) Open-circuit voltage amplitude $\left(V_{O C}\right)$ in frequency at acceleration level of $1.0 \mathrm{~g}$.

Fig. 14: Micrograph of the micro-fabricated piezoelectric transducer (PT) with its electrode split into four regions and its frequency response.

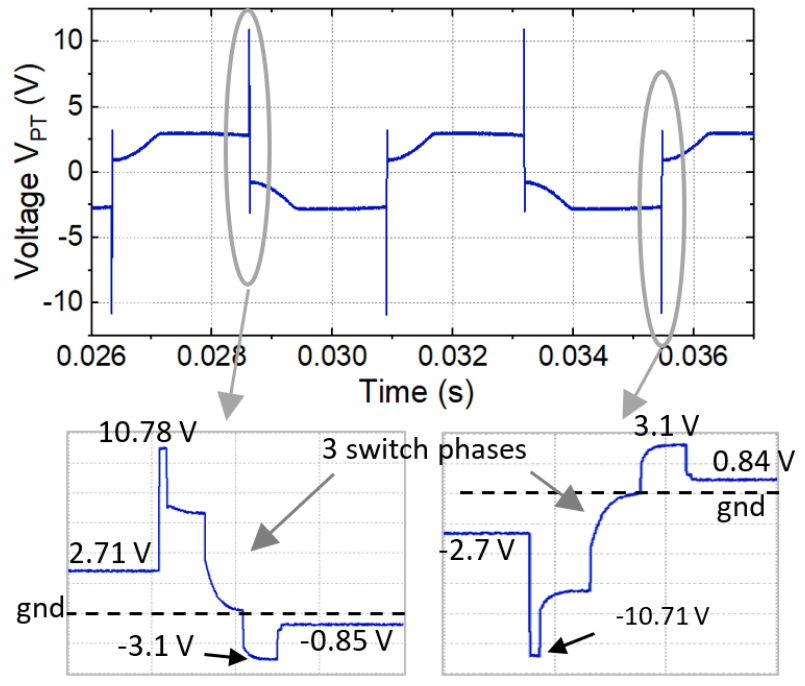

(a) $1 \mathrm{SC}$ enabled.

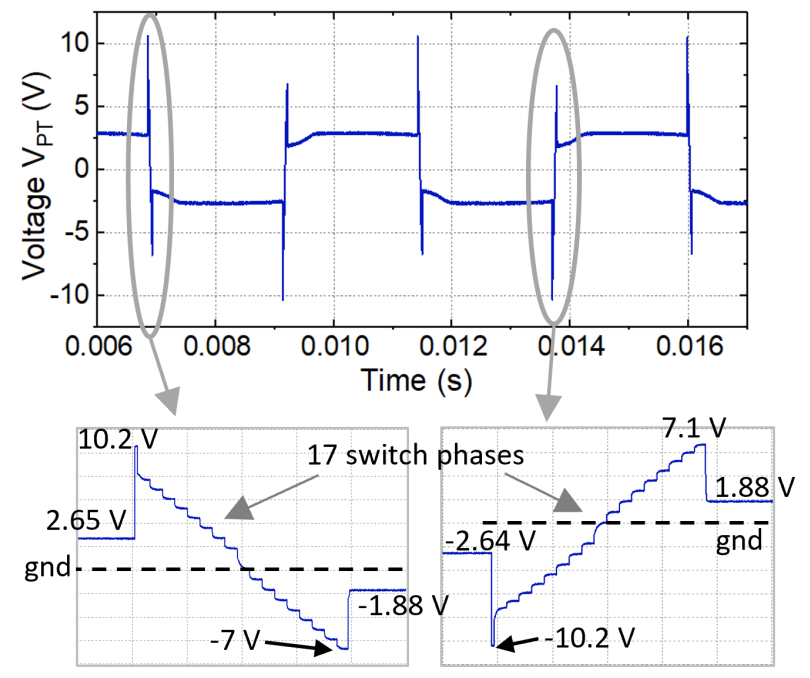

(b) 8 SCs enabled.

Fig. 15: Measured waveform of voltage $V_{P T}$.

the test chip is shown in Fig. 13. To meet the high-voltage requirement, an extra mask has been used in the $0.18 \mu \mathrm{m}$ CMOS process to add high-voltage thick-oxide transistors.

In order to evaluate the proposed system, a MEMS PT with its electrode split into 4 regions was fabricated and the micrograph is shown in Fig. 14a. The PT was fabricated in a commercial MEMS foundry, MEMSCAP, using AlN as the piezoelectric material. Fig. 14b shows the open-circuit voltage amplitude $\left(V_{O C}\right)$ by sweeping the excitation frequency around its natural frequency at a base acceleration level of $1.0 \mathrm{~g}$. The total capacitance with the 4 regions connected in parallel is measured to be $C_{P}=1.94 \mathrm{nF}$. The capacitance is reduced to $155 \mathrm{pF}$ while the 4 regions are connected in series and this value is in the operational range of the on-chip SC arrays. Hence, the on-chip SC arrays should be configured to form an equivalent capacitor of around $155 \mathrm{pF}$. To achieve this, the switches D4, D1 and D0 in Fig. 12 are ON while the other switches are kept OFF. This results in an equivalent capacitor of around $152 \mathrm{pF}$. During the measurements with the proposed SE-SSHC rectifier, a shaker (LDS V406) was excited at the natural frequency of the MEMS PT at $219 \mathrm{~Hz}$ and driven by a sine wave from a function generator (Agilent 33250A) amplified by a power amplifier (LDS PA100E).

Fig. 15 shows the measured waveforms of the voltage across the PT, $V_{P T}$, while the number of enabled on-chip SCs are set to 1 and 8 . It can be seen that there are spikes for voltage flip instants due to the increased $V_{P T}$ voltage (increased by 4 times) due to the series connection of the four electrode regions. In Fig. 15a, only one SC in the proposed rectifier is enabled. The zoom-in voltage flip instants for $V_{P T}$ flipped from positive to negative and from negative to positive are also shown in the two sub-figures. Before the voltage flip commences, the four regions are configured to a series connection and it can be observed that the voltage $V_{P T}$ is increased by 4 times. Then the voltage flip commences and $V_{P T}$ is flipped in three phases. Since there is only one SC enabled, three switch pulses are generated to perform the voltage flip. After the voltage is flipped, the four electrode 


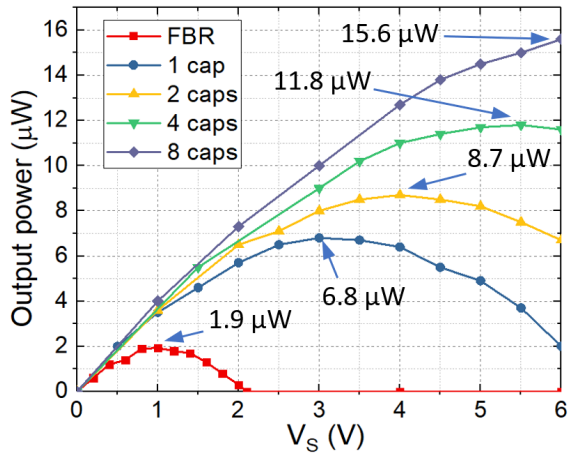

(a)

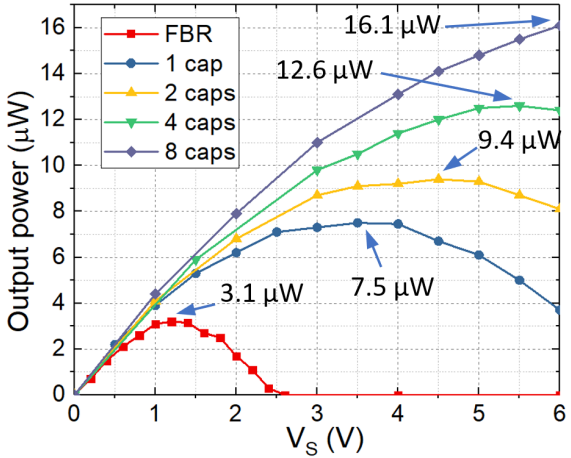

(b)

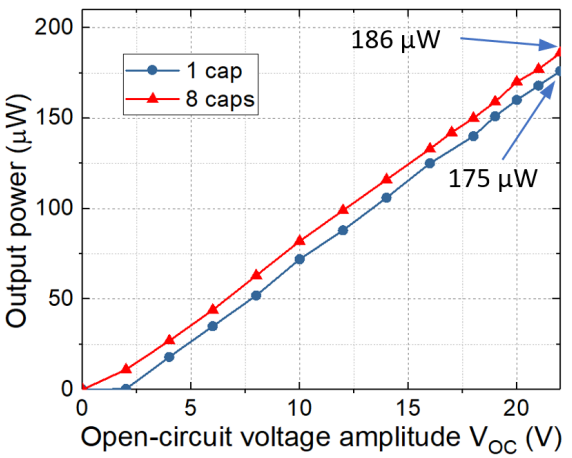

(c)

Fig. 16: Measured output power of a FBR and the proposed SE-SSHC rectifier with up to 8 on-chip SCs enabled. (a) Output power with on-chip diodes of $V_{D} \approx 0.25 \mathrm{~V}$ while $V_{O C}=2.5 \mathrm{~V}$. (b) Output power with off-chip diodes of $V_{D} \approx 0.05 \mathrm{~V}$ while $V_{O C}=2.5 \mathrm{~V}$. (c) Output power in a wide range of excitation levels (up to $V_{O C}=22 \mathrm{~V}$, equivalent to $3.4 \mathrm{~g}$ ) with $V_{S}=5 \mathrm{~V}$.

TABLE II: Performance comparison with state-of-the-art interface circuits.

${ }^{(a)}$ with on-chip diodes. ${ }^{(b)}$ with off-chip diodes.

\begin{tabular}{cccccccc}
\hline \hline Reference & Technique & PT & $\begin{array}{c}\text { Piezoelectric } \\
\text { capacitance }\end{array}$ & Frequency & $V_{O C}$ & Inductor & $\frac{P_{I C}}{P_{F B R}}$ \\
\hline \hline JSSC2010 [6] & $\begin{array}{c}\text { Bias-flip } \\
\text { (SSHI) }\end{array}$ & $\begin{array}{c}\text { Mide } \\
\text { V22B }\end{array}$ & $18 \mathrm{nF}$ & $225 \mathrm{~Hz}$ & 2.4 & $820 \mu \mathrm{H}$ & 4 \\
\hline JSSC2012 [23] & PSCE & $\begin{array}{c}\text { Mide } \\
\text { V22B }\end{array}$ & $19.5 \mathrm{nF}$ & $173 \mathrm{~Hz}$ & $9 \mathrm{~V}$ & $10 \mathrm{mH}$ & 2.1 \\
\hline JSSC2014 [24] & $\begin{array}{c}\text { Energy- } \\
\text { investing }\end{array}$ & $\begin{array}{c}\text { Mide } \\
\text { V22B }\end{array}$ & $15 \mathrm{nF}$ & $143 \mathrm{~Hz}$ & $2.6 \mathrm{~V}$ & $330 \mu \mathrm{H}$ & 3.6 \\
\hline JSSC2014 [14] & SSHI & $\begin{array}{c}\text { Custom } \\
\text { MEMS }\end{array}$ & $8.5 \mathrm{nF}$ & $155 \mathrm{~Hz}$ & $8.2 \mathrm{~V}$ & $470 \mu \mathrm{H}$ & 2.5 \\
\hline TPEL2016 [9] & SECE & $\begin{array}{c}\text { Q220- } \\
\text { A4303YB }\end{array}$ & $52 \mathrm{nF}$ & $60 \mathrm{~Hz}$ & $2.35 \mathrm{~V}$ & $560 \mu \mathrm{H}$ & 3 \\
\hline ISSCC2016 [25] & SSHI & $\begin{array}{c}\text { MIDE } \\
\text { V21B }\end{array}$ & $26 \mathrm{nF}$ & $134 \mathrm{~Hz}$ & $2.45 \mathrm{~V}$ & $3.3 \mathrm{mH}$ & 6.8 \\
\hline JSSC2017 [17] & FCR & $\begin{array}{c}\text { Piezo } \\
\text { P5A4E }\end{array}$ & $0.08 \mathrm{nF}$ & $110 \mathrm{kHz}$ & - & $\begin{array}{c}\text { On-chip } \\
\text { caps }\end{array}$ & 4.8 \\
\hline This work & SE-SSHC & Custom MEMS & $1.94 \mathrm{nF}$ & $219 \mathrm{~Hz}$ & $2.5 \mathrm{~V}$ & On-chip caps & $\begin{array}{l}3.6-8.2^{(\mathrm{a})} \\
2.4-5.2^{(\mathrm{b})}\end{array}$ \\
\hline \hline
\end{tabular}

regions is configured back to a parallel connection. The results shows that the voltage flip efficiency is around $31 \%$. The waveform while all the $8 \mathrm{SCs}$ are enabled is shown in Fig. $15 \mathrm{~b}$ and in this case, 17 pulses are needed to flip the voltage. The voltage flip efficiency in this case is measured to be around $71 \%$.

Fig. 16 shows the measured output power transferred into the capacitor $C_{S}$ connected at the output of the FBR. In Fig. 16a, the excitation level is fixed at a level such that the open-circuit zero-to-peak voltage is $V_{O C}=2.5 \mathrm{~V}$. The output voltage $V_{S}$ is varied from $0 \mathrm{~V}$ to $6 \mathrm{~V}$ to show the maximum power points for all the cases. The voltage drop of the onchip Schottky diodes forming the FBR is measured at around $V_{D} \approx 0.25 \mathrm{~V}$. The output power values of a FBR and the proposed SE-SSHC rectifier with 1, 2, 4 and 8 SCs enabled are measured and plotted. When using the on-chip FBR, it can be seen that the peak output power extracted by a passive FBR is $1.9 \mu \mathrm{W}$. After the SE-SSHC rectifier is turned ON with one $\mathrm{SC}$ enabled, the peak power is increased to $6.8 \mu \mathrm{W}$ with $3.58 \times$ performance enhancement. This is because the voltage $V_{P T}$ is synchronously flipped and the flip efficiency is measured to be around $31 \%$. While all the 8 on-chip SCs are enabled, the peak power achieves $15.6 \mu \mathrm{W}$ and the performance is enhanced by $8.2 \times$ compared with the FBR. A FBR built of four offchip Schottky diodes is also used during measurement, as shown in Fig. 16b, and the diode voltage drop in this case is measured at around $V_{D} \approx 0.05 \mathrm{~V}$. Due to the lower voltage drop, the extracted power by a passive FBR is significantly increased. Although the power from the proposed SE-SSHC rectifier is increased as well, the performance improvement compared to the FBR is decreased due to the lower $V_{D}$. The peak power values marked in Fig. 16a and Fig. 16b correspond to specific $V_{S}$ values. In order to achieve power at these optimal points, maximum power point tracking (MPPT) technique is required to dynamically adjust $V_{S}$ according to input excitation levels [11], [26]. Fig. 16c shows the measured output power while the excitation level is varied from $0 \mathrm{~g}$ to $3.4 \mathrm{~g}$, which is equivalent to $V_{O C}$ voltage varying from $0 \mathrm{~V}$ 
to $22 \mathrm{~V}$. The reason of not keeping increasing the excitation level is because this is the highest level that the MEMS PT can tolerate before fracture occurs. The measurement shows the highest possible power that the MEMS PT can provide and this value is measured to be $186 \mu \mathrm{W}$ with the proposed SE-SSHC rectifier while 8 on-chip SCs are enabled.

Table II shows the performance comparisons between the proposed SE-SSHC rectifier and reported interface circuits for piezoelectric vibration energy harvesting. Among all the previously reported rectifiers, most of them require large inductors to perform voltage flip, which significantly increases the system size. Although [17] proposed a flipping-capacitor rectifier (FCR) achieving voltage flip with on-chip capacitors, this particular rectifier aims to work with PTs with extremely small $C_{P}$ capacitance. The proposed SE-SSHC rectifier achieves high performance enhancement without using any inductors. In addition, thanks to the split-electrode (SE) design, the proposed rectifier can serve PTs with much larger $C_{P}$ capacitance using small on-chip $\mathrm{SC}$ values to perform voltage flip.

\section{CONCLUSION}

This paper presents a SE-SSHC rectifier for piezoelectric energy harvesting which performs synchronous voltage flip using on-chip SCs. Compared with the conventional SSHC rectifier, the proposed circuit employs a PT with its electrode split into several regions and these regions are temporarily connected in series during voltage flip instants. With this topology, the effective capacitance of the PT is significantly decreased; hence, the required SCs for a SSHC rectifier are also significantly decreased such that they can be implemented on-chip. Compared with state-of-the-art rectifiers using inductors, the proposed circuit is fully integrated, which dramatically decreases the system volume, especially preferable for miniaturized energy harvesting systems. Compared with the recently reported FCR circuit, the proposed system can work with a wide variety of PTs with inherent capacitance values ranging from 10 's $\mathrm{pF}$ to 1 's $\mathrm{nF}$.

\section{REFERENCES}

[1] S. Du and A. A. Seshia, "A fully integrated split-electrode synchronizedswitch-harvesting-on-capacitors (se-sshc) rectifier for piezoelectric energy harvesting with between 358enhancement," in 2018 IEEE International Solid - State Circuits Conference - (ISSCC), Conference Proceedings, pp. 152-154.

[2] G. Zhou, L. Huang, W. Li, and Z. Zhu, "Harvesting ambient environmental energy for wireless sensor networks: A survey," Journal of Sensors, vol. 2014, p. 20, 2014. [Online]. Available: http://dx.doi.org/10.1155/2014/815467

[3] P. D. Mitcheson, E. M. Yeatman, G. K. Rao, A. S. Holmes, and T. C. Green, "Energy harvesting from human and machine motion for wireless electronic devices," Proceedings of the IEEE, vol. 96, no. 9, pp. 14571486, 2008.

[4] A. Khaligh, Z. Peng, and Z. Cong, "Kinetic energy harvesting using piezoelectric and electromagnetic technologies - state of the art," IEEE Transactions on Industrial Electronics, vol. 57, no. 3, pp. 850-860, 2010.

[5] G. D. Szarka, B. H. Stark, and S. G. Burrow, "Review of power conditioning for kinetic energy harvesting systems," Power Electronics, IEEE Transactions on, vol. 27, no. 2, pp. 803-815, 2012.

[6] Y. K. Ramadass and A. P. Chandrakasan, "An efficient piezoelectric energy harvesting interface circuit using a bias-flip rectifier and shared inductor," IEEE Journal of Solid-State Circuits, vol. 45, no. 1, pp. 189 204, 2010 .
[7] S. Du, Y. Jia, C. D. Do, and A. A. Seshia, "An efficient sshi interface with increased input range for piezoelectric energy harvesting under variable conditions," IEEE Journal of Solid-State Circuits, vol. 51, no. 11, pp. $2729-2742,2016$.

[8] P. Gasnier, J. Willemin, S. Boisseau, G. Despesse, C. Condemine, G. Gouvernet, and J. J. Chaillout, "An autonomous piezoelectric energy harvesting ic based on a synchronous multi-shot technique," IEEE Journal of Solid-State Circuits, vol. 49, no. 7, pp. 1561-1570, 2014.

[9] M. Dini, A. Romani, M. Filippi, and M. Tartagni, "A nanopower synchronous charge extractor ic for low-voltage piezoelectric energy harvesting with residual charge inversion," IEEE Transactions on Power Electronics, vol. 31, no. 2, pp. 1263-1274, 2016.

[10] H. Shen, H. Ji, J. Qiu, Y. Bian, and D. Liu, "Adaptive synchronized switch harvesting: A new piezoelectric energy harvesting scheme for wideband vibrations," Sensors and Actuators A: Physical, vol. 226, pp. 21-36, 2015.

[11] M. Shim, J. Kim, J. Jeong, S. Park, and C. Kim, "Self-powered 30uw to $10 \mathrm{mw}$ piezoelectric energy harvesting system with $9.09 \mathrm{~ms} / \mathrm{v}$ maximum power point tracking time," IEEE Journal of Solid-State Circuits, vol. 50, no. 10 , pp. 2367-2379, 2015

[12] G. Shi, Y. Xia, Y. Ye, L. Qian, and Q. Li, "An efficient self-powered synchronous electric charge extraction interface circuit for piezoelectric energy harvesting systems," Journal of Intelligent Material Systems and Structures, p. 1045389X15624796, 2016.

[13] S. Du, Y. Jia, and A. A. Seshia, "An efficient inductorless dynamically configured interface circuit for piezoelectric vibration energy harvesting," IEEE Transactions on Power Electronics, vol. 32, no. 5, pp. 3595$3609,2017$.

[14] E. E. Aktakka and K. Najafi, "A micro inertial energy harvesting platform with self-supplied power management circuit for autonomous wireless sensor nodes," IEEE Journal of Solid-State Circuits, vol. 49, no. 9, pp. 2017-2029, 2014.

[15] A. Romani, M. Filippi, and M. Tartagni, "Micropower design of a fully autonomous energy harvesting circuit for arrays of piezoelectric transducers," Power Electronics, IEEE Transactions on, vol. 29, no. 2, pp. 729-739, 2014.

[16] D. A. Sanchez, J. Leicht, F. Hagedorn, E. Jodka, E. Fazel, and Y. Manoli, "A parallel-sshi rectifier for piezoelectric energy harvesting of periodic and shock excitations," IEEE Journal of Solid-State Circuits, vol. 51, no. 12, pp. 2867-2879, 2016.

[17] Z. Chen, M. K. Law, P. I. Mak, W. H. Ki, and R. P. Martins, "Fully integrated inductor-less flipping-capacitor rectifier for piezoelectric energy harvesting," IEEE Journal of Solid-State Circuits, vol. 52, no. 12, pp. 3168-3180, 2017.

[18] S. Du and A. A. Seshia, "An inductorless bias-flip rectifier for piezoelectric energy harvesting," IEEE Journal of Solid-State Circuits, vol. 52, no. 10, pp. 2746-2757, 2017.

[19] Y. Yang and L. Tang, "Equivalent circuit modeling of piezoelectric energy harvesters," Journal of Intelligent Material Systems and Structures, 2009.

[20] S. Du, G. A. J. Amaratunga, and A. A. Seshia, "A cold-startup sshi rectifier for piezoelectric energy harvesters with increased open-circuit voltage," IEEE Transactions on Power Electronics, pp. 1-1, 2018.

[21] L. Wu, X. D. Do, S. G. Lee, and D. S. Ha, "A self-powered and optimal sshi circuit integrated with an active rectifier for piezoelectric energy harvesting," IEEE Transactions on Circuits and Systems I: Regular Papers, vol. 64, no. 3, pp. 537-549, 2017.

[22] S. Du, Y. Jia, C. Zhao, G. A. J. Amaratunga, and A. A. Seshia, "A passive design scheme to increase the rectified power of piezoelectric energy harvesters," IEEE Transactions on Industrial Electronics, vol. 65 , no. 9, pp. 7095-7105, 2018.

[23] T. Hehn, F. Hagedorn, D. Maurath, D. Marinkovic, I. Kuehne, A. Frey, and Y. Manoli, "A fully autonomous integrated interface circuit for piezoelectric harvesters," IEEE Journal of Solid-State Circuits, vol. 47, no. 9, pp. 2185-2198, 2012.

[24] D. Kwon and G. A. Rincon-Mora, "A single-inductor 0.35 um cmos energy-investing piezoelectric harvester," IEEE Journal of Solid-State Circuits, vol. 49, no. 10, pp. 2277-2291, 2014.

[25] D. A. Sanchez, J. Leicht, E. Jodka, E. Fazel, and Y. Manoli, "A 4uw-to$1 \mathrm{mw}$ parallel-sshi rectifier for piezoelectric energy harvesting of periodic and shock excitations with inductor sharing, cold start-up and up to 681extraction improvement," in 2016 IEEE International Solid-State Circuits Conference (ISSCC), Conference Proceedings, pp. 366-367.

[26] N. Kong and D. S. Ha, "Low-power design of a self-powered piezoelectric energy harvesting system with maximum power point tracking," Power Electronics, IEEE Transactions on, vol. 27, no. 5, pp. 2298-2308, 2012. 


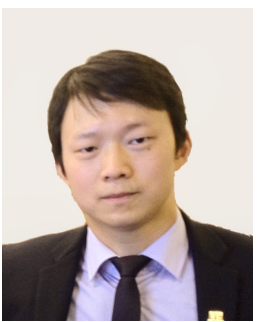

Sijun Du (S'14-M'17) received a First Class in B.Eng degree in electrical engineering from University Pierre and Marie Curie (UPMC), Paris, France, in 2011, a Distinction in M.Sc degree in electrical and electronics engineering from Imperial College, London, U.K., in 2012. He started his Ph.D research in Oct 2014 and obtained the Ph.D degree in engineering from University of Cambridge, Cambridge, U.K. in 2017. He joined the Berkeley Wireless Research Center (BWRC), Department of Electrical Engineering and Computer Sciences (EECS) at University of California at Berkeley, Berkeley, CA, 94720, U.S.A. in April 2018, where he is currently a post-doctoral researcher in communication systems and circuits.

He worked at the Laboratory LIP6 of University Pierre Marie Curie, Paris, and then worked as a digital IC engineer in Shanghai between 2012 and 2014 He was with the Cambridge Nanoscience Centre at University of Cambridge for his Ph.D research between 2014 and 2017. He was an engineer intern at Qualcomm Technology Inc., San Diego, California, U.S.A. between August 2016 and November 2016. He was a visiting scholar at the Brain-Chip Research Center (BCRC), Department of Microelectronics, Fudan University, Shanghai, China, between December 2017 and March 2018.

His research interests include energy harvesters and associated interfaces, energy conversion system and circuits, power electronics, power management circuits, rectification circuits, wireless communication circuits.

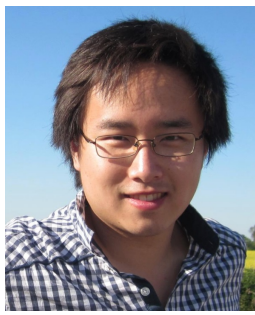

Yu Jia (S'13-M'14) received a First Class (Honours) in MEng Electromechanical Engineering from the University of Southampton in 2010, and $\mathrm{PhD}$ in Engineering from the University of Cambridge in 2014. He was then a Research Associate at Cambridge for a year. He is currently a Senior Lecturer in Mechanical Engineering at the University of Chester, leads the Smart Microsystems Research Group. His research interests include vibration energy harvesting, microelectromechanical systems (MEMS), nonlinear vibration dynamics and smart integrated systems. He is a co-founder of 8power Ltd. and is a steering board member of the Energy Harvesting Network.

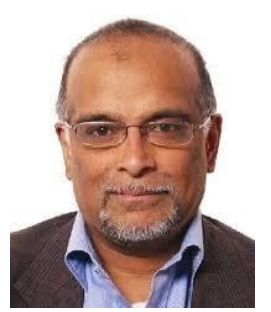

Gehan A. J. Amaratunga received his BSc degree in Electronic Engineering and the IEE Prize for the best graduating student from Cardiff University, UK (1979) and $\mathrm{PhD}$ degree from University of Cambridge in 1983. Since receiving his $\mathrm{PhD}$ from Cambridge, he has held other academic and research positions at Southampton University, the University of Liverpool and Stanford University. He is a Professor of Engineering and Head of Electronics, Power and Energy Conversion at the University of Cambridge, Chief of Research \& Innovation, Sri Lanka Institute of Nanotechnology (SLINTEC) and Visiting Professor, Nanyang Technological University, Singapore. He is a Fellow of the Royal Academy of Engineering. He is also a Fellow of the Royal Society of Arts and the Institution of Engineering and Technology (formerly the IEE). He has a long record of successful collaborations with industry partners in Europe, the USA and Asia (Philips, Infineon, Ford, Motorola, Toshiba, Fuji Electric, Samsung, Intel, Nokia, Dyson). He is the founder of six start-up companies in technology, Cambridge Semiconductor - CamSemi (acquired by Power Integrations inc. 2015), Enecsys (acquired by Solar City inc. 2015), Wind Technologies, Nanoinstruments (acquired by Aixtron AG 2007), Camutronics and Zinergy, which have collectively attracted in excess of $\$ 150 \mathrm{M}$ in venture capital investment and been successful in transforming advanced research to commercially successful products. In 2007 he was awarded the Silver Medal by the Royal Academy of Engineering for Outstanding personal contributions to British engineering which has resulted in commercial success. He sits on the Investment Advisory Board of NES Partners, a Danish venture capital fund. He has also acted as an expert in patent litigation for multinationals and as an adviser to leading investment firms. He has published over 600 archived academic papers (h-index 80) and is an inventor on 44 granted patents. His research is in the broad area of materials and technologies for electrical energy and power. It intersects electrical and electronic engineering with chemistry, physics, materials science and information systems.

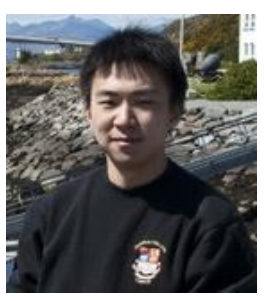

Chun Zhao (S'14-M'16) received the B.Eng. degree in measurement and control technology and instrument from the Huazhong University of Science and Technology, Wuhan, China, in 2009; the M.Sc. degree in analog and digital IC design from Imperial College London, London, U.K., in 2011; and the Ph.D. degree in microelectromechanical systems (MEMS) from the University of Southampton, Southampton, U.K., in 2016.

From April 2015 to March 2016, he was a full time Research Scientist at Sharp Laboratories of Europe, Oxford, U.K., working on the research and development of MEMS acoustic devices and integrated control circuit design based on TFT. He joined the Department of Engineering, University of Cambridge in April 2016, where he is currently a Research Associate in MEMS. He has authored or co-authored more than 20 journal and conference publications in the field of MEMS sensor design, interface circuit design and MEMS energy harvesters. He has also served as an invited reviewer for journals including IEEE JMEMS, Elsevier Sensors and Actuators A: Physical, MDPI Sensors and Nature Microsystems \& Nanoengineering.

His current research interests include MEMS, microresonators, miniature sensors (such as inertial sensors) and actuators (including ultrasonic devices), energy harvesters, MEMS system modelling and circuit design.

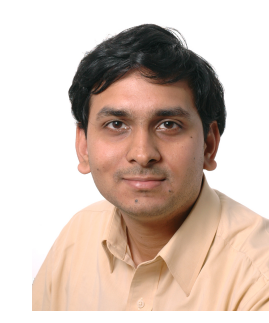

Ashwin A. Seshia (S'97-M'02-SM'10) received his BTech in Engineering Physics from IIT Bombay in 1996 and his MS and PhD degrees in Electrical Engineering and Computer Science from the University of California Berkeley in 1999 and 2002 respectively. He is presently the Professor of Microsystems Technology at Cambridge University. He is also a Fellow of Queens' College and a co-investigator of the Cambridge Centre for Smart Infrastructure and Construction. His research interests are in the domain of micro- and nano-engineered dynamical systems with applications to sensors and sensor systems. He is a Fellow of the Institute of Physics and a Fellow of the Institution of Engineering and Technology. Ashwin serves on the editorial boards of the IEEE Journal of Microelectromechanical systems and the IEEE Transactions on Ultrasonics, Ferroelectrics and Frequency Control. 